\title{
STABLE ABSOLUTELY UBIQUITOUS STRUCTURES
}

\author{
A. A. IVANOV
}

(Communicated by Andreas R. Blass)

\begin{abstract}
It is proved that absolutely ubiquitous structures with trivial algebraic closures are monadically stable.
\end{abstract}

Let $L$ be a finite language, and let $M$ be an $L$-structure with countably infinite domain. The age of $M$, denoted by $\mathscr{J}(M)$, is the set of all isomorphism types of finite substructures of $M$. A model $M$ is absolutely ubiquitous if $M$ is uniformly locally finite and every countable locally finite $L$-structure with age $\mathscr{J}(M)$ is isomorphic to $M$. It is known that an absolutely ubiquitous structure is countably categorical and model-complete. It is not known if an absolutely ubiquitous structure has stable or superstable theory. In [1-3] a classification was given for absolutely ubiquitous structures over relational languages. Such structures are finitely partitioned. This will be proved here in a slightly more general case using completely different techniques.

Notation. $B<M$ if $B$ is a substructure of $M$,

$$
\operatorname{Str}(B) \text { is the substructure of } M \text { generated by } B \text {. }
$$

\section{Lemma of E. A. Palyutin}

The following lemma is a simple generalization of an old result of E. A. Palyutin on categorical universal theories [4].

Lemma 1. A countable structure $M$ is absolutely ubiquitous iff

(1) $M$ is uniformly locally finite; and

(2) there is a function $s: \omega \rightarrow \omega$ such that for every $n$, for every substructure $B$ of $M$, and for every type $p\left(y, b_{0}, \ldots, b_{n-1}\right)$ over $\left\{b_{0}, \ldots, b_{n-1}\right\} \subseteq B$, if $B$ contains all isomorphism types of $s(n)$-generated substructures of $M$ then there is $b \in B$ such that $M \vDash p\left(b, b_{0}, \ldots, b_{n-1}\right)$.

Proof. Let $M$ be absolutely ubiquitous. We construct $s$ by induction. Suppose $s$ is defined for all $i<n$. Suppose $R_{0}, \ldots, R_{t}$ (and $T_{0}, \ldots, T_{l}$ ) are complete formulas which determine all complete $n$-types $((n+1)$-types $)$ over $\varnothing\left(\operatorname{Th}(M)\right.$ is $\omega$-categorical). By model-completeness we may suppose that $R_{i}$

Received by the editors March 26, 1992 and, in revised form, August 7, 1992.

1991 Mathematics Subject Classification. Primary 03C45, 03C35.

Key words and phrases. Stability, absolutely ubiquitous structures.

(c) 1994 American Mathematical Society $0002-9939 / 94 \$ 1.00+\$ .25$ per page 
are $\forall$-formulas and $T_{j}$ are $\exists$-formulas. We define the set $G_{k}^{i, j}$ as follows. Let

$$
\boldsymbol{\Phi}_{i, j}=\forall x_{0}, \ldots, x_{n-1}\left(R_{i}\left(x_{0}, \ldots, x_{n-1}\right) \rightarrow \exists y\left(T_{j}\left(y, x_{0}, \ldots, x_{n-1}\right)\right) .\right.
$$

If $M \vDash \Phi_{i, j}$ let $G_{k}^{i, j}$ be

$\mathrm{Th}_{\forall}(M) \cup\left\{\right.$ there are $z_{1}, \ldots, z_{m}$ which generate substructure embedding all types of $k$-generated substructures of $M$ \}

$$
\cup\left\{\neg \Phi_{i, j}\right\} .
$$

If $M \vDash \neg \Phi_{i, j}$ let $G_{k}^{i, j}$ be $\forall x(x \neq x)$,

If for all $k$ the set $G_{k}^{i, j}$ is consistent then there is a countable model $N$ of the theory $\operatorname{Th}_{\forall}(M)$ with $\mathscr{J}(N)=\mathscr{J}(M)$ and $N \vDash \neg \Phi_{i, j}$. It is a contradiction.

Let

$$
\begin{gathered}
k(i, j)=\min \left(k: G_{k}^{i, j} \text { is inconsistent }\right), \\
s(n)=\max (k(i, j): 0 \leq i \leq t, 0 \leq j \leq l) .
\end{gathered}
$$

Now if $B$ and $p$ are as in (2), formula $R_{i}$ determines $\operatorname{tp}\left(\left\langle b_{0}, \ldots, b_{n-1}\right\rangle\right)$ and formula $T_{j}$ determines type $p\left(y, x_{0}, \ldots, x_{n-1}\right)$, then since $G_{s(n)}$ is inconsistent we have

$$
B \vDash \forall x_{0}, \ldots, x_{n-1}\left(R_{i}\left(x_{0}, \ldots, x_{n-1}\right) \rightarrow \exists y T_{j}\left(y, x_{0}, \ldots, x_{n-1}\right)\right) .
$$

Since $R_{i}$ is universal, there is $b \in B$ such that $B \vDash T_{j}\left(b, b_{0}, \ldots, b_{n-1}\right)$, and since $T_{j}$ is $\exists$-formula, $M \vDash T_{j}\left(b, b_{0}, \ldots, b_{n-1}\right)$.

If (1) and (2) hold in $M$ then $M$ is countably saturated. If for countable $N$ we have $\mathscr{J}(N)=\mathscr{J}(M)$ then $N$ is isomorphic to a substructure of $M$. By back-and-forth argument using (2) we can prove $M$ is isomorphic to $N$.

\section{Structures With A Finite NUMBER OF TERM FUNCTIONS}

We suppose here that there are terms $d_{1}, \ldots, d_{t}$ such that for every term $d$ the corresponding function under essential variables is one of the functions defined by $d_{1}, \ldots, d_{t}$ in $M$. For example, a uniformly locally finite unary algebra is such a structure.

Proposition 1. Let $M$ be an absolutely ubiquitous structure with a finite number of term functions. Then the theory of $M$ is stable.

Proof. It is sufficient to prove that $\operatorname{Th}(M)$ has not the independence property because by [5] $\operatorname{Th}(M)$ has not the strict order property. For a contradiction suppose that there are a formula $F(\mathbf{x}, \mathbf{y})$ and sequences $\left\langle\mathbf{a}_{i}: i \in \omega\right\rangle$ and $\left\langle\mathbf{c}_{\sigma}: \sigma \in 2^{\omega}\right\rangle$ such that $F F\left(\mathbf{a}_{i}, \mathbf{c}_{\sigma}\right)$ iff $\sigma(i)=0$. For ease of notation suppose that $\mathbf{a}_{i}$ and $\mathbf{c}_{\sigma}$ are singletons. Assume terms $d_{1}, \ldots, d_{t}$ depend on at most $m$ variables. Let $B$ be a finite substructure of $M$ containing parameters of $F$ and all types of $s(2 m+2)$-generated substructures of $M$. We may suppose that $\mathbf{a}_{1}, \ldots, \mathbf{a}_{2 m+2}$ is a sequence of order indiscernibles over $B$, and by Lemma 1 suppose

$$
\left\{\mathbf{c}_{\sigma}: \sigma \in 2^{2 m+2}\right\} \subseteq \operatorname{Str}\left(B \cup\left\{\mathbf{a}_{1}, \ldots, \mathbf{a}_{2 m+2}\right\}\right)
$$

(of course, we neglect parameters). Define $\sigma \in 2^{2 m+2}$ by $\sigma(i)=0$ iff $i$ is odd. We know that for some $\mathbf{a}_{j_{1}}, \ldots, \mathbf{a}_{j_{k}}(k \leq m)$ and $\mathbf{b} \subseteq B$ and term $d_{i}$, $\mathbf{c}_{\sigma}=d_{i}\left(\mathbf{b}, \mathbf{a}_{j_{1}}, \ldots, \mathbf{a}_{j_{k}}\right)$. There are $\mathbf{a}_{2 n-1}$ and $\mathbf{a}_{2 n}(n \leq m+1)$ not among 
$\mathbf{a}_{j_{1}}, \ldots, \mathbf{a}_{j_{k}}$. By indiscernibility, $\vDash F\left(\mathbf{a}_{2 n-1}, \mathbf{c}_{\sigma}\right)$ iff $\vDash F\left(\mathbf{a}_{2 n}, \mathbf{c}_{\sigma}\right)$, which is a contradiction.

\section{Structures With trivial ClOSURE}

A structure $M$ has trivial closure if $\operatorname{acl}(X \cup Y)=\operatorname{acl}(X) \cup \operatorname{acl}(Y)$ holds in $M$. For example, absolutely ubiquitous structures with unary terms only have trivial closure. Indeed, by Lemma 1 there is some type of finite substructures of $M$ such that for every $B$ of this type $\operatorname{acl}(X \cup Y)$ is in the substructure generated by $X \cup Y \cup B$. Since every term is unary, we have $\operatorname{acl}(X \cup Y) \subseteq$ $\operatorname{acl}(X) \cup \operatorname{acl}(Y) \cup B$. We can choose $B$ with $B \cap \operatorname{acl}(X \cup Y) \subseteq \operatorname{acl}(\varnothing)$. So, $\operatorname{acl}(X \cup Y)=\operatorname{acl}(X) \cup \operatorname{acl}(Y)$.

By $[1,6]$ absolutely ubiquitous structures in a relational language are monadically stable. By the description of countably categorical monadically stable structures [6], every absolutely ubiquitous monadically stable structure has trivial closure. It is not hard to find an absolutely ubiquitous structure with a finite number of term functions which does not have trivial closure. So, the situation of Proposition 1 is quite different than that of [1-3]. But in the case of trivial closure we have the following

Proposition 2. Absolutely ubiquitous structures with trivial closure are monadically stable.

Proof. Let us prove that the relation $x \in \operatorname{acl}(y)$ on nonalgebraic elements is an equivalence relation. If $a \in \operatorname{acl}(b)$ and $b \notin \operatorname{acl}(a)$ then there is some type of finite substructures of our model $M$ such that for every $B$ of this type some realization of $\operatorname{tp}(b \mid a)$ is in the substructure generated by $B \cup\{a\}$. By triviality of closure it is in $\operatorname{acl}(B)$ and so $a \in \operatorname{acl}(B)$. But we can choose $\operatorname{acl}(B)$ so that $\operatorname{acl}(B) \cap\{a\} \subseteq \operatorname{acl}(\varnothing)$. We have now $a \in \operatorname{acl}(\varnothing)$.

Let us prove that Shelah's $R$-rank of $M$ is one. Let $\mathbf{a}_{i}, i \in \omega$, be an infinite sequence, and suppose the set of nonalgebraic complete formulas $F\left(x, \mathbf{a}_{i}\right)$ is $n$ inconsistent. Choose a finite $B<M$ containing all types of $s\left(\ln \left(\mathbf{a}_{i}\right)\right)$-generated substructures of $M$. By Lemma $1 \operatorname{Str}\left(B \cup\left\{\mathbf{a}_{i}: i \in \omega\right\}\right)$ contains realizations of all formulas $F\left(x, \mathbf{a}_{i}\right)$. Of course, the corresponding realization of formula $F\left(x, \mathbf{a}_{i}\right)$ is in $\operatorname{Str}\left(B \cup \mathbf{a}_{i}\right)$ and by triviality of closure it is in $\operatorname{acl}(B)$. This contradicts $n$-inconsistency of $\left\{F\left(x, \mathbf{a}_{i}\right): i \in \omega\right\}$.

Since $\omega$-categorical superstable theory is $\omega$-stable, the Morley rank of $M$ is one. There is $E_{0} \in F E(\varnothing)$ with strongly minimal classes. Let $G=\{\operatorname{acl}(a)-$ $\operatorname{acl}(\varnothing): a$ is nonalgebraic $\}$. For $X_{1}, X_{2} \in G$ let $\left\langle X_{1}, X_{2}\right\rangle \in E_{1}$ iff $X_{1}, X_{2}$ meet the same $E_{0}$-classes. Hence $E_{1}$ is a finite equivalence relation.

If $a$ and $b$ are in the same $E_{0}$-class and $c$ is a finite sequence disjoint to $(\operatorname{acl}(a) \cup \operatorname{acl}(b))-\operatorname{acl}(\varnothing)$ then $\operatorname{tp}(a \mid c)=\operatorname{tp}(b \mid c)$. Indeed otherwise for some formula $H(x, \mathrm{c}), M \vDash H(a, \mathrm{c}) \& \neg H(b, \mathrm{c})$. For the corresponding strongly minimal type $p(x, \mathbf{d})$ (consisting of the $E_{0}$-class with $a$ and $b$ ) we have that, for example, $p(x$, d $) \& H(x$, c) is algebraic. Since acl is trivial and $x \in \operatorname{acl}(y)$ is an equivalence relation, $a \in \operatorname{acl}(\varnothing)$. This is a contradiction.

We now have a 0 -definable equivalence relation $E$ on $M$ with finite classes such that $M / E$ is finitely partitioned: $E$ is $\operatorname{acl}(x)=\operatorname{acl}(y), E_{1}$-classes on $M / E-\operatorname{acl}(\varnothing) / E$ are infinite, $E_{1}$ is invariant under $\operatorname{Aut}(M)$, and for every such $E_{1}$-class $Q$ the pointwise stabilizer of $M / E-Q$ in $\operatorname{Aut}(M)$ induces 
$\operatorname{Sym} Q)$. By [6] $M$ is monadically stable ( $M$ is cellular in the notation of Schmerl [2]).

Proposition 2 yields an easy proof of the main result of [1]. It is enough to note that in a relational language $\operatorname{acl}(a)=\operatorname{acl}(\varnothing) \cup\{a\}$. But this is an easy corollary of Lemma 1.

\section{REFERENCES}

1. I. M. Hodkinson and H. D. Macpherson, Relational structures induced by their finite induced substructures, J. Symbolic Logic 53 (1988), 222-230.

2. J. Schmerl, Coinductive $\omega$-categorical theories, J. Symbolic Logic 55 (1990), 1130-1137.

3. A. H. Lachlan, Complete theories with only universal and existential axioms, J. Symbolic Logic 52 (1987), 698-711.

4. E. A. Palyutin, Modeli so schetno kategorichnymi universalnymi teoriami, Algebra i Logika 10 (1971), 23-32.

5. H. D. Macpherson, Absolutely ubiquitous structures and $\omega$-categorical groups, Quart. J. Math. Oxford (2) 39 (1988), 483-500.

6. A. H. Lachlan, $\omega$-categorical tree-decomposable structures, preprint, 1990.

Mathematical Institute, University of Wroclaw, Pl. Grunwaldzki $2 / 4,50-384$ Wroclaw, Poland

E-mail address: logicuwr@plwruw11.bitnet 